\title{
Measurements of multi-particle correlations and collective flow with the ATLAS detector
}

\author{
Tomasz Bold ${ }^{* \dagger}$ \\ AGH University of Science and Technology, Krakow, Poland \\ E-mail: tomasz.boldefis.agh.edu.pl
}

\begin{abstract}
The measurement of flow harmonics of charged particles from $v_{2}$ to $v_{7}$ in $\mathrm{Pb}+\mathrm{Pb}$ collisions in the wide range of transverse momentum and pseudorapidity provides not only a way to study the initial state of the nuclear collisions and soft particle collective dynamics, but also provides insight into jet quenching via the measurement of flow harmonics at high transverse momenta. The longitudinal fluctuations of $v_{n}$ and event-plane angles $\Psi_{n}$ are also presented. The longitudinal flow decorrelations have contributions from $v_{n}$-magnitude fluctuations and event-plane twist. A four-particle correlator is used to separate these two effects. Results show that both effects have a linear dependence on pseudorapidity separation from $v_{2}$ to $v_{5}$, and show a small but measurable variation with collision energy. While collectivity is well established in collisions involving heavy nuclei, its evidence in $p p$ collisions is less clear. In order to assess the collective nature of multiparticle production, the correlation measurements are extended to include azimuthal correlations measured using multi-particle cumulants. The measurements of multi-particle cumulants $c_{2}\{2$ $8\}$ confirm the evidence for collective phenomena in $p+\mathrm{Pb}$ and low-multiplicity $\mathrm{Pb}+\mathrm{Pb}$ collisions. For $p p$ collisions, the measurements of cumulants do not yet provide clear evidence for collectivity as they are susceptible to event-by-event multiplicity fluctuations. A new modified cumulant method, which suppresses both the contribution of multiplicity fluctuations and non-flow effects, is used to address this issue.
\end{abstract}

The European Physical Society Conference on High Energy Physics

5-12 July, 2017

Venice

\footnotetext{
*Speaker.

${ }^{\dagger}$ On behalf of the ATLAS Collaboration
} 


\section{Introduction}

The correlation studies in ATLAS [1] concentrate on detailed analysis of the QGP dynamics in $\mathrm{Pb}+\mathrm{Pb}$ collisions. Smaller systems of $p+\mathrm{Pb}$ or $p p$ collisions are also in the area of interest where an unquestioned QGP signature has still to be found.

\section{Correlation studies in $\mathbf{P b}+\mathbf{P b}$}

The charged-hadrons azimuthal flow coefficients of up to $v_{7}$ are measured in $\mathrm{Pb}+\mathrm{Pb}$ data using the scalar product (SP) and two-particle correlations (2PC) methods [2]. The measurement is performed in a wide range of transverse momenta, $p_{\mathrm{T}}$ for $0-80 \%$ most central events with the emphasise on the most central collisions. Non-zero values are found for up to $v_{7}$ in the intermediate $p_{\mathrm{T}}$ range as shown in Figure 1. Results obtained with 2PC and SP methods are consistent. The integrated $v_{n}$ harmonics vary little with centrality except for the $v_{2}$ which maximises at mid-centralities due to the sensitivity to the initial-state eccentricities.
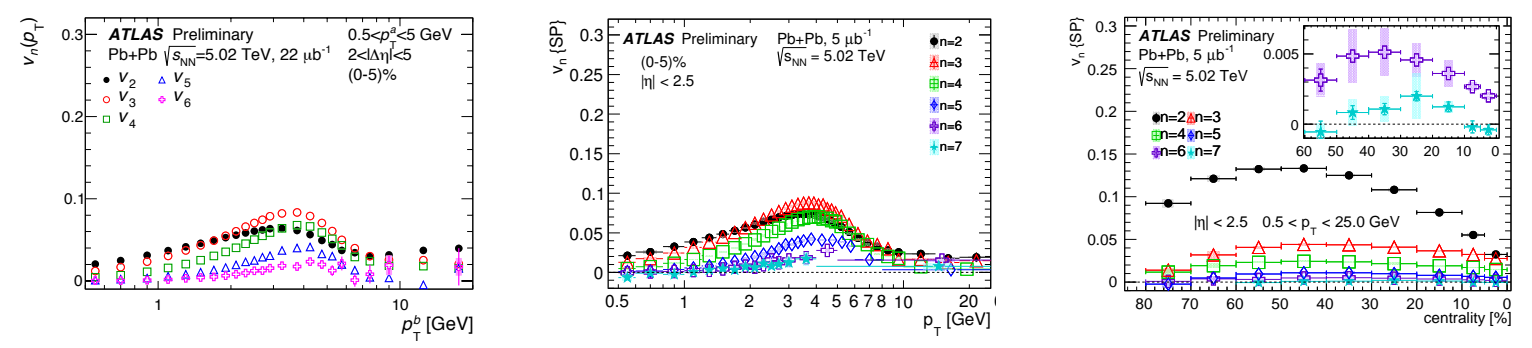

Figure 1: The $v_{n}$ as a function of $p_{\mathrm{T}}$ measured with 2PC (left), SP methods (center) and integrated over the $p_{\mathrm{T}}$ range of $0.5-25 \mathrm{GeV}$ and pseudorapidity $|\eta|<2.5$ (left) as a function of collision centrality in $\mathrm{Pb}+\mathrm{Pb}$ collisions at $\sqrt{s_{\mathrm{NN}}}=5.02 \mathrm{TeV}[2]$.

In the $\mathrm{Pb}+\mathrm{Pb}$ collisions the flow longitudinal decorrelation is studied [3]. The correlators sensitive the flow $v_{n}$ magnitude $r_{n \mid n ; k}$ and event-plane twist $R_{n \mid n ; 2}$ are measured as a function of pseudorapidity, $\eta$. The decorrelation of the magnitude shown in Figure 2 for $v_{2}$ and $v_{3}$ increases with $\eta$ indicating breaking of 2PC factorisation to 1-particle correlation with $\eta$. The effect has different dynamics as a function of centrality for $v_{2}$ and $v_{3}$. The $r_{n \mid n ; k}$ remains centrality independent for the latter. The effect is weaker at higher center-of-mass energies. The $R_{n \mid n ; 2}$ correlators, shown in Figure 3, show a similar magnitude and behaviour with $\eta$ and energy indicating that the eventplane twist has an equally significant contribution to the longitudinal $v_{n}$ dynamics.

\section{Small system measurements}

\subsection{Sub-event cumulants}

The observation of the ridge structure in $p p$ collisions at the LHC [4] opened the discussion on the presence of collectivity in small collision systems or a different origin of the observed phenomena. It was found that a method robust against local correlations due to jets or resonances has to be established to resolve the puzzle. For instance in the cumulants measurement not only 

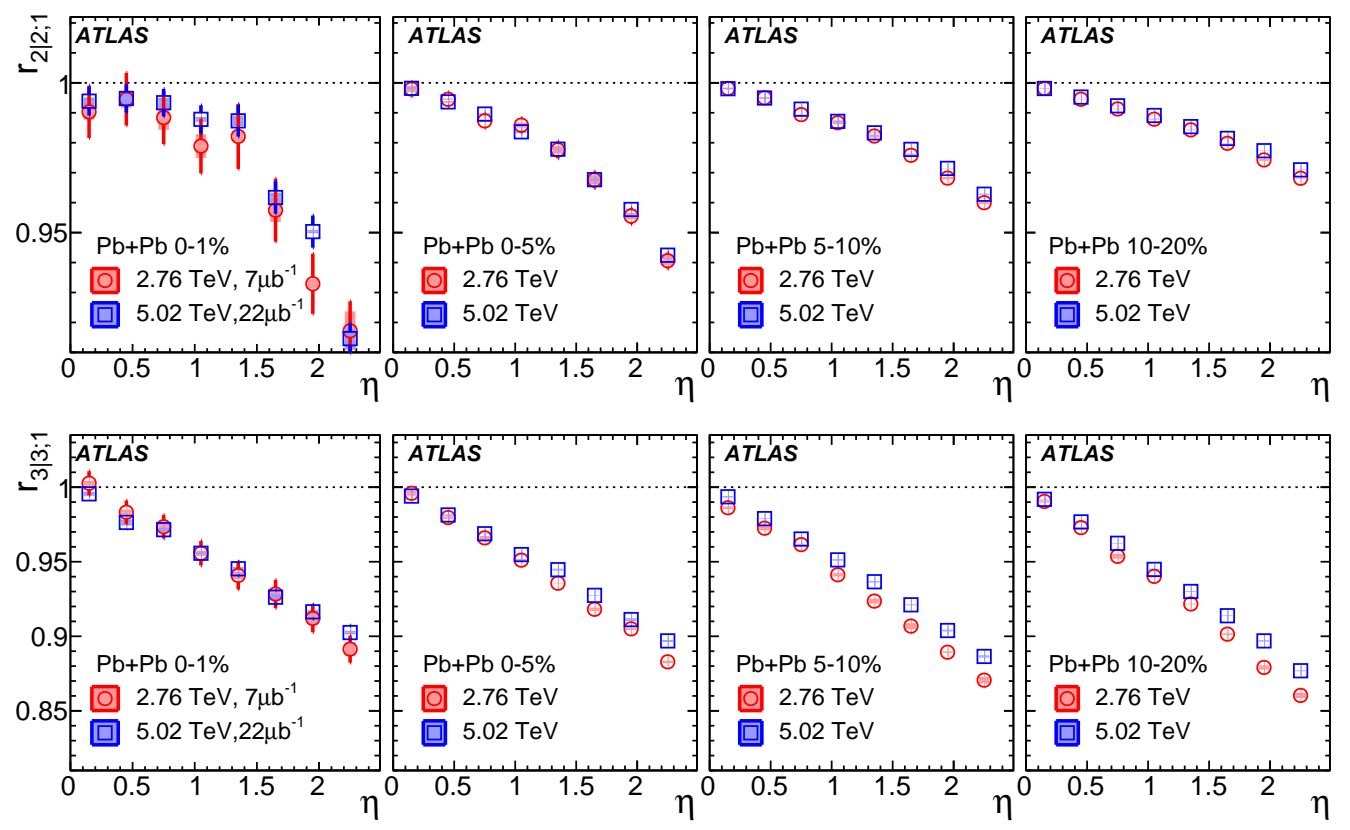

Figure 2: The $r_{n \mid n ; k}$ correlator as a function of $\eta$ at two energies for $n=2$ (top) and $n=3$ (bottom) in bins of centrality [3].
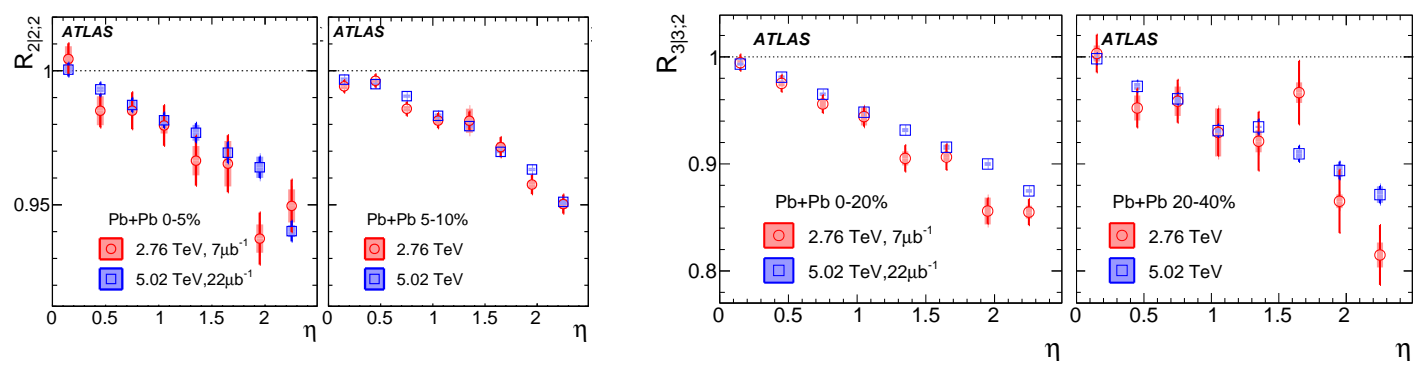

Figure 3: The $R_{n \mid n ; 2}$ correlator as a function of $\eta$ at two energies for $n=2$ (left) and $n=3$ (right) in bins of centrality [3].

the quantitative but also qualitative results may depend on an arbitrary choice of kinematics of reference particles [5]. A novel method of sub-event cumulants is devised to reduce the non-flow background on measured correlations [6]. In this method the event is partitioned into sub-events (two or three in case of $p p$ measurements) and particles from disparate sub-events are used. In practice, the formulation of traditional cumulants is modified as follows:

$$
\begin{gathered}
\left\langle\{2\}_{n}\right\rangle=e^{i n\left(\phi_{1}-\phi_{2}\right)} \rightarrow\left\langle\{2\}_{n}\right\rangle_{a \mid b}=e^{i n\left(\phi_{1}^{a}-\phi_{2}^{b}\right)} \\
\left\langle\{4\}_{n}\right\rangle=e^{i n\left(\phi_{1}+\phi_{2}-\phi_{3}-\phi_{4}\right)} \rightarrow\left\langle\{4\}_{n}\right\rangle_{2 a \mid b, c}=e^{i n\left(\phi_{1}^{a}+\phi_{2}^{a}-\phi_{3}^{b}-\phi_{4}^{c}\right)} \\
c_{n}\{4\}=\left\langle\left\langle\{4\}_{n}\right\rangle\right\rangle-2\left\langle\left\langle\{2\}_{n}\right\rangle\right\rangle^{2} \rightarrow c_{n}^{2 a \mid b, c}\{4\}=\left\langle\left\langle\{4\}_{n}\right\rangle\right\rangle_{2 a \mid b, c}-2\left\langle\left\langle\{2\}_{n}\right\rangle\right\rangle_{a \mid b}\left\langle\left\langle\{2\}_{n}\right\rangle\right\rangle_{a \mid c}
\end{gathered}
$$

where the particles are taken from three ranges of pseudorapidity into which the full detector cover- 
age $|\eta|<2.5$ is split. The angle brackets denote an event average while double angel brackets denote averaging over the event and then over many events. The flow coefficient, $v_{n}\{4\}=\sqrt[4]{-c_{n}\{4\}}$, is then only defined when the cumulant $c_{n}\{4\}$ assumes positive values while positive cumulant is an indicator of lack of collectivity.

In the ATLAS measurement [7], a cross-check of this method on the $p+\mathrm{Pb}$ collision system is performed as shown in the left panel of Figure 4 where the results obtained from the standard cumulant, two sub-events or three sub-events methods are consistent except for very peripheral collisions where only three sub-events method indicates collectivity $\left(c_{2}\{4\}<0\right)$. The results of the method application to the $p p$ system is shown in the right panel of Figure 4 where the three subevents method indicates presence of non-local correlation. The comparison of the $v_{n}$ measurements obtained with the 2PC method and three sub-event is shown in Figure 5 for the $p p$ collisions at two energies as well as for $p+\mathrm{Pb}$. Flow at the level of $4 \%$ independent of the multiplicity is observed in all conditions. In comparison to the $2 \mathrm{PC}$ measurements deploying various techniques of subtracting non-flow contributions estimated from peripheral collisions the three sub-events results yield smaller $v_{2}$. The $v_{3}$ in $p p$ system is found to be consistent with zero.
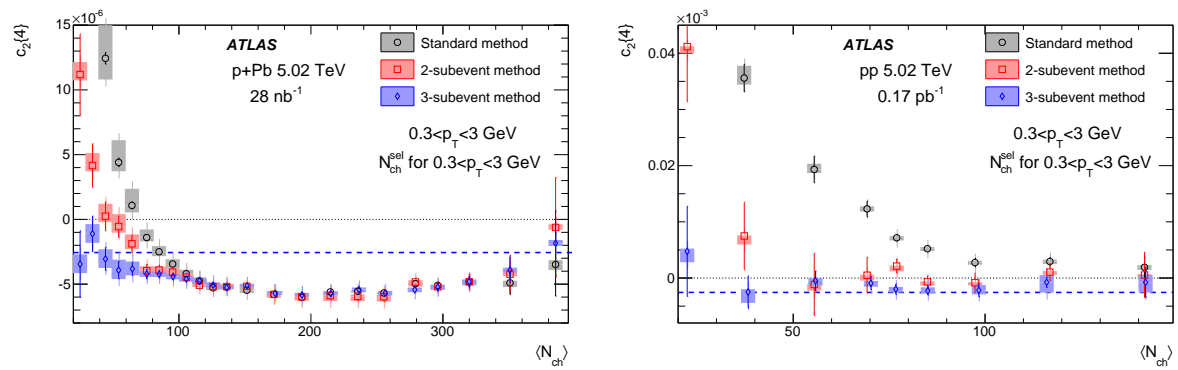

Figure 4: The $c_{2}\{4\}$ measured with the standard, two sub-events and three sub-event methods in $p+\mathrm{Pb}$ collisions (left) and pp collisions (right) [7].

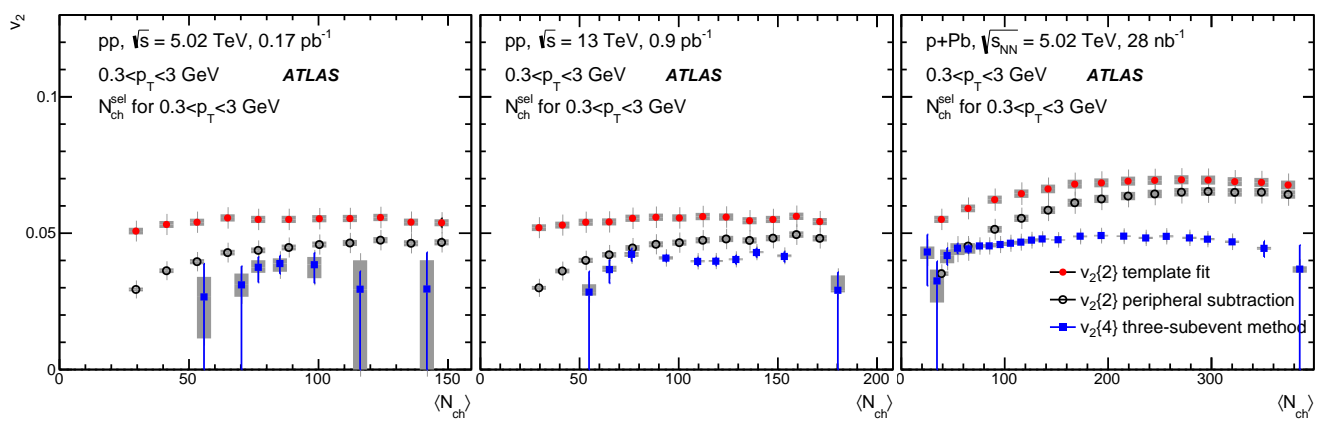

Figure 5: The $v_{2}$ measured in $p p$ at $\sqrt{s}=5.02 \mathrm{TeV}$ (left), at $13 \mathrm{TeV}$ (centre) and $p+\mathrm{Pb}$ at $\sqrt{s_{\mathrm{NN}}}=5.02 \mathrm{TeV}$ (right) with a sub-event cummulant method as well as wiht 2PC methods with two non-flow removal procedures based on peripheral collisions [7].

\section{Summary}

In these proceedings ATLAS correlation measurements in $\mathrm{Pb}+\mathrm{Pb}, p+\mathrm{Pb}$ and $p p$ collision systems are presented. ATLAS measures flow harmonics up to $v_{7}$ in a broad range of $p_{\mathrm{T}}$. The lon- 
gitudinal decorrelations are studied in $\mathrm{Pb}+\mathrm{Pb}$ at 2.76 and $5.02 \mathrm{TeV}$. It is found that the slope of this decorrelation is slightly bigger at lower energies. Both, the flow magnitude change and the event-plane twist influence the decorrelation at a similar level. For small collision systems a novel method of sub-event cumulants is proposed in order to robustly eliminate non-flow effects. The $v_{2}$ coefficient is found to be about $4 \%$ in a wide range of event activity quantified by charged particle multiplicity in $p p$ as well as in $p+\mathrm{Pb}$ collisions.

\section{Acknowledgements}

This work was supported in part by the National Science Center grant UMO-2015/19/B/ST2/00906 and by PL-Grid Infrastructure.

\section{References}

[1] ATLAS Collaboration, JINST 3 (2008) S08003.

[2] ATLAS Collaboration, ATLAS-CONF-2016-105, https://cds.cern.ch/record/2220372

[3] ATLAS Collaboration, arXiv:1709.02301 [nucl-ex].

[4] CMS Collaboration, JHEP 1009 (2010) 091 [arXiv:1009.4122 [hep-ex]].

[5] ATLAS Collaboration, Eur. Phys. J. C 77 (2017) no.6, 428 [arXiv:1705.04176 [hep-ex]].

[6] J. Jia, M. Zhou and A. Trzupek, arXiv:1701.03830 [nucl-th].

[7] ATLAS Collaboration, arXiv:1708.03559 [hep-ex]. 